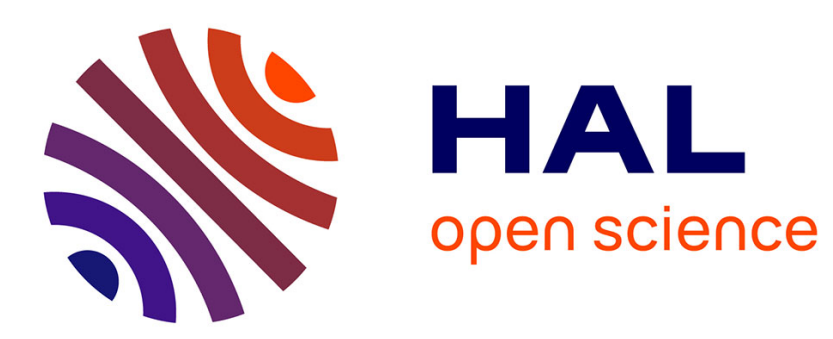

\title{
Flory approximation for directed branched polymers and directed percolation
}

\author{
T.C. Lubensky, J. Vannimenus
}

\section{To cite this version:}

T.C. Lubensky, J. Vannimenus. Flory approximation for directed branched polymers and directed percolation. Journal de Physique Lettres, 1982, 43 (11), pp.377-381. 10.1051/jphyslet:019820043011037700 . jpa-00232062

\section{HAL Id: jpa-00232062 https://hal.science/jpa-00232062}

Submitted on 1 Jan 1982

HAL is a multi-disciplinary open access archive for the deposit and dissemination of scientific research documents, whether they are published or not. The documents may come from teaching and research institutions in France or abroad, or from public or private research centers.
L'archive ouverte pluridisciplinaire HAL, est destinée au dépôt et à la diffusion de documents scientifiques de niveau recherche, publiés ou non, émanant des établissements d'enseignement et de recherche français ou étrangers, des laboratoires publics ou privés. 
Classification

Physics Abstracts

$36.20-61.40 \mathrm{~K}-05.90$

\title{
Flory approximation for directed branched polymers and directed percolation
}

\author{
T. C. Lubensky (*) and J. Vannimenus \\ Groupe de Physique des Solides de l'Ecole Normale Supérieure, \\ 24, rue Lhomond, 75231 Paris Cedex 05, France
}

(Reçu le 17 mars 1982, accepté le 14 avril 1982)

\begin{abstract}
Résumé. - Nous généralisons l'approche due à Flory pour le rayon de giration des polymères en solution diluée à des systèmes ayant une orientation privilégiée. Le rayon moyen augmente alors avec la taille $N$ comme $N^{v_{\|}}$dans cette direction, et comme $N^{v_{\perp}}$ dans les directions transverses. Dans le cas des polymères branchés dirigés (animaux sur réseau) et de la percolation dirigée, la dimension critique supérieure $d_{\mathrm{c}}$ est prédite correctement et les valeurs obtenues pour $v_{\|}$et $v_{\perp}$ sont en très bon accord avec celles données par d'autres méthodes, en dimensions 2 et 3 . Le développement en $\left(d_{c}-d\right)$ des exposants montre que les résultats ne sont pas exacts en général, comme pour les polymères non orientés.

Abstract. - The Flory approximation for the radius of gyration of dilute polymers is generalized to systems with a preferred direction. The mean radius then increases with their size $N$ as $N^{v_{11}}$ in the preferred direction and as $N^{v_{\perp}}$ in the transverse directions. In the case of directed branched polymers (lattice animals) and of directed percolation, the upper critical dimension $d_{\mathrm{c}}$ is predicted correctly, and the values obtained by $v_{\|}$and $v_{\perp}$ are in very good agreement with the values from other methods in dimensions 2 and 3 . An expansion in $\left(d_{c}-d\right)$ shows that the results are not exact in general, as is the case for unoriented polymers.
\end{abstract}

The Flory approximation [1] for the exponent $v$ relating the degree of polymerization $N$ to the mean end to end separation, $R$, of a linear polymer is extremely good for all spatial dimensions $d$ between 2 and 4 . Recently, this approximation has been extended with remarkable success to lattice animals (branched polymers) and percolation [2]. In the former case, the result $v=5 / 2(d+2)$ gives the correct upper critical dimension [3] $d_{\mathrm{c}}=8$ and the exact result [4] $v=1 / 2$ for $d=3$. In addition, the value of $v=5 / 8$ for $d=2$ is very close to the best numerical value [5] $v=0.6408 \pm 0.008$, and the values of $v$ for $3<d<8$ are very close to those obtained by various numerical methods $[6,7]$.

In this paper, we generalize the Flory approximation to situations such as those encountered in directed percolation [8-11] and directed animals [12-15] in which there is a privileged direction,

(*) Permanent address : Department of Physics, University of Pennsylvania, Philadelphia PA 19104, U.S.A. 
$\hat{e}$, with different correlation length exponents $v_{\|}$and $v_{\perp}$ for directions parallel and perpendicular to $\hat{e}$. In the Flory approximation, a competition between an elastic energy $F_{\mathrm{el}}$ and a repulsive energy $F_{\text {rep }}$ arising from repulsive interactions between monomers determines $v . F_{\text {el }}$ is predominantly entropic and tends to keep the radius of gyration, $R$, at its value in the absence of monomerinteractions. Its expression is taken from that for a Gaussian chain :

$$
F_{\mathrm{el}} \cong R^{2} / R_{0}^{2}
$$

with $R_{0} \sim N^{v_{0}}$ where $v_{0}=1 / 2$ and $1 / 4$ for linear and branched polymers respectively. $F_{\text {rep }}$ is determined by short range 2 -monomer encounters. In dilute solutions, it is approximated by

$$
F_{\text {rep }} \sim N^{2} / R^{d} .
$$

In high dimensions, $F_{\text {rep }}$ is negligible and the polymer remains Gaussian with $v=v_{0}$ in spite of the interactions. When $F_{\text {rep }}$ becomes of order one, the competition between $F_{\text {el }}$ and $F_{\text {rep }}$ becomes important. This leads to an upper critical dimension $d_{\mathrm{c}}=2 / v_{0}$ and

$$
v=2\left(1+v_{0}\right) /(d+2)
$$

for $d<d_{\mathrm{c}}$.

For directed systems, we introduce a transverse radius $R_{\perp}$ and a longitudinal radius $R_{\|}$, which behave as $N^{v_{\perp}}$ and $N^{v_{11}}$ respectively. The elastic energy then takes the form

$$
F_{\mathrm{el}} \sim\left(\frac{R_{\|}}{R_{\|}^{0}}\right)^{2}+\left(\frac{R_{\perp}}{R_{\perp}^{0}}\right)^{2}
$$

with $R_{\|}^{0} \sim N^{v_{1}^{0},} R_{\perp}^{0} \sim N^{v_{\perp}^{0}}$, and the Gaussian exponents are given by $v_{\perp}^{0}=v^{0}, v_{\|}^{0}=2 v^{0}$. The repulsion energy is inversely proportional to the volume of the polymer and becomes

$$
F_{\text {rep }} \sim \frac{N^{2}}{R_{\|} R_{\perp}^{d-1}} .
$$

The upper critical dimensionality is determined by requiring $F_{\text {rep }}\left(R_{\|}^{0}, R_{\perp}^{0}\right) \sim 1$. This yields

$$
\left(d_{c}-1\right) v_{\perp}^{0}+v_{\|}^{0}=2 \text {. }
$$

Below $d_{\mathrm{c}}$, the total energy is to be minimized with respect to both $R_{\|}$and $R_{\perp}$. This gives :

$$
v_{\|}-v_{\perp}=v_{\|}^{0}-v_{\perp}^{0}
$$

and

$$
v_{\perp}=\frac{2+3 v_{\perp}^{0}-v_{\|}^{0}}{d+2}
$$

For branched polymers, $v_{\perp}^{0}=1 / 4, v_{\|}^{0}=1 / 2$ and $d_{c}=7$ from equation (6), in agreement with field-theory calculations [12]. Below $d_{\mathrm{c}}$, the exponents are given by :

$$
v_{\perp}=\frac{9}{4(d+2)}, \quad v_{\|}=\frac{11+d}{4(d+2)} .
$$


These results may be compared to the expansions obtained within the field-theory approach, at first order in $\varepsilon=7-d$ :

$$
\begin{aligned}
& \left.v_{\perp} \text { (Flory) }=\frac{1}{4}(1+\varepsilon / 9)=v_{\perp} \text { (F.Th. }\right) \\
& \left.\left.v_{\|} \text {(Flory }\right)=\frac{1}{2}(1+\varepsilon / 18)<v_{\|} \text {(F.Th. }\right)=\frac{1}{2}(1+\varepsilon / 12) .
\end{aligned}
$$

For lower dimensions, the expressions (9) may be compared with results obtained for directed lattice animals. For $d=2$, equations (9) give :

$$
v_{\perp}=9 / 16=0.5625, \quad v_{\|}=13 / 16=0.8125
$$

whereas the numerical results [13-15] suggest that

$$
\begin{aligned}
& v_{\perp}=1 / 2 \text { (presumably exact) } \\
& v_{\|}=0.818 \pm 0.001 .
\end{aligned}
$$

The agreement is satisfactory, in view of the simplicity of the argument, but not quite as good as for the isotropic case. For $d=3$, one obtains :

$$
v_{\perp}=9 / 20, \quad v_{\|}=7 / 10
$$

whereas a direct enumeration method [13] gives :

$$
v_{\perp}=0.45 \pm 0.005, \quad v_{\|}=0.70 \pm 0.005
$$

a remarkable agreement.

The case of directed percolation is somewhat more complicated, and we follow the derivation given by Isaacson and Lubensky [3] for isotropic percolation. Percolation may be viewed as a gelation problem for branched polymers, and the interaction energy is reduced by a screening factor $N_{\mathrm{w}}$ related to the weight average degree of polymerization :

$$
F_{\text {rep }} \sim \frac{N^{2}}{R_{\|} R_{\perp}^{d-1}} \frac{1}{N_{\mathrm{w}}} .
$$

In principle, $N_{\mathrm{w}}$ depends on the dimension below $d_{\mathrm{c}}$, but it is a slowly varying function and at the present level of approximation it is reasonable to assume that it keeps its mean-field value :

$$
N_{\mathrm{w}} \sim N^{1 / 2}
$$

which is the same result as for the isotropic case [3]. The upper critical dimensionality is then reduced to :

$$
\left(d_{\mathrm{c}}-1\right) v_{\perp}^{0}+v_{\|}^{0}=3 / 2
$$

and $d_{\mathrm{c}}=5$, since we are dealing with branched polymers. The minimization of the free energy yields :

$$
\begin{aligned}
v_{\|}-v_{\perp} & =1 / 4 \\
v_{\perp} & =\frac{7}{4(d+2)} .
\end{aligned}
$$


These expressions have been derived for gelation, and the corresponding exponents for percolation are obtained simply by multiplying them by the gap exponent $\Delta$ [3]. Scaling expressions relate $\Delta$ to other percolation exponents :

$$
\Delta=\beta+\gamma=\frac{1}{2}\left[(d-1) v_{\perp}^{(p)}+v(p)+\gamma\right]
$$

so its value can be inferred from published values for these exponents. At first order in $\varepsilon=(5-d)$, field-theory calculations show that $[9,11]$

$$
\begin{gathered}
v_{\perp}^{(p)}=\frac{1}{2}\left(1+\frac{\varepsilon}{8}\right) ; \quad v(p)=1+\frac{\varepsilon}{12} \\
\Delta=2+0\left(\varepsilon^{2}\right)
\end{gathered}
$$

and the predictions of equations (13) and (14) are :

$$
\begin{aligned}
& v_{\perp}^{(p)}=\Delta v_{\perp}=\frac{1}{2}(1+\varepsilon / 7) \\
& v \|^{(p)}=\Delta v_{\|}=1+\varepsilon / 14 .
\end{aligned}
$$

As usual, the Flory approximation is wrong by a small amount in that limit.

In two and three dimensions, we can obtain the values of the directed percolation exponents from accurate results obtained for Reggeon field-theory, since an exact mapping exists between both problems [11]. These read, for $d=2$ :

$$
\begin{aligned}
v^{(p)} & =1.736 \pm 0.001 \\
v_{\perp}^{(p)} & =1.104 \pm 0.007 \\
\Delta & =2.563 \pm 0.006 .
\end{aligned}
$$

(Note that the notations of reference [11] are slightly different from ours : in particular $v=v(p)$, $z=2 v_{\perp}^{(p)} / v_{\|}^{(p)}$ and $\eta=1-\eta_{\|}$, with $\gamma=\left(2-\eta_{\|}\right) v_{\|}^{(p)}$.)

Using this value for $\Delta$, the Flory predictions are :

$$
v_{\|}^{(p)}=1.762 \pm 0.004 ; \quad v_{\perp}^{(p)}=1.121 \pm 0.003
$$

and the agreement is striking, though not perfect.

For $d=3$, the field-theory gives [11] :

$$
\begin{aligned}
v(p) & =1.27 \pm 0.01 \\
v_{\perp}^{(p)} & =0.735 \pm 0.015 \\
\Delta & =2.16 \pm 0.025
\end{aligned}
$$

whereas equations (13) and (14) lead to

$$
v(p)=1.295 \pm 0.015 ; \quad v_{\perp}^{(p)}=0.756 \pm 0.008
$$

The confidence intervals of both sets of values overlap in that case, but the numerical results are less accurate.

In principle equations (6)-(8) could be applied to linear polymers with inherent anisotropies. For example a dipolar polymer (i.e. one with alternating positive and negative charges along the chain) in an external electric field will be elongated along the direction of the field. Alternately 
one might consider a self-avoiding walk on a lattice in which a fraction $p$ of the steps must be in the positive direction on the lattice. Evidently if $p=1$, self-avoidance is automatically insured since there are no steps in the negative direction. In this case the Gaussian exponents $v_{\|}^{0}=1$ and $v_{\perp}^{0}=1 / 2$ are obtained. For $p<1$, however, self-repulsion could have some effects. A naive application of equations (6) and (8) yields $d_{\mathrm{c}}=3, v_{\perp}=5 / 2(d+2)$ and $v_{\|}=1 / 2[(d+7) /(d+2)]$. $d_{c}=3$ agrees with perturbation theory and $\varepsilon$-expansion calculations [16] which show infrared singularities for $d \leqslant 3$. The interpretation of $v_{\|}$is uncertain, however, since it is greater than one for all $d<3$; and we are disinclined at the moment to take the Flory approximation for this problem seriously.

It appears likely [12] that $v(d)$ for directed animals in dimension $d$ is identical to $v(d+1)$ for normal animals in dimension $(d+1)$. If this is the case, it would be better to take for $v_{\perp}$ the Flory value for $v(d+1)$ :

$$
v_{\perp}(d)=v(d+1)=\frac{5}{2(d+3)}
$$

rather than equation (9). This approximation has the advantage that it gives the correct value for $v_{\perp}$ at $d=7$ and the apparently exact value $v_{\perp}(2)=1 / 2$ for $d=2$. For $d=3$ it yields $v_{\perp}=5 / 12$ in less spectacular agreement with the numerical value of $v_{\perp}=0.45$ than the prediction of equation (9).

Acknowledgments. - We are grateful to J. des Cloizeaux for discussions regarding the problem of dipolar polymers.

Note added in proof. - In a recent preprint (to be published in J. Phys. A Letters), Redner and Coniglio have independently derived exactly the same results presented in this paper. In addition, they consider the Flory theory for a directed branched polymer in a $\theta$-solvent.

\section{References}

[1] FloRy, P., Principles of polymer chemistry, Chap. XII (Cornell University Press, Ithaca-N.Y.) 1971. De Gennes, P. G., Scaling concepts in polymer physics, Chap. I (Cornell University Press, IthacaN.Y.) 1979.

[2] Isaacson, J. and Lubensky, T. C., J. Physique-Lett. 41 (1980) L-469.

[3] Lubensky, T. C. and IsaAcson, J., Phys. Rev. A 20 (1979) 2130.

[4] Parisi, G. and Sourlas, N., Phys. Rev. Lett. 43 (1979) 744.

[5] Derrida, B. and de Seze, L., J. Physique 43 (1982) 475.

[6] Lubensky, T. C. and McKane, A. J., J. Physique-Lett. 42 (1981) L-331.

[7] De Alcantara Bonfim, O. F., Kirkham, J. E. and McKane, A. J., J. Phys. A (Math. Gen.) 13 (1980) L 247.

[8] Blease, J., J. Phys. C (Sol. State Phys.) 10 (1977) 917.

[9] Obukhov, S. P., Physica 101A (1980) 145.

[10] Kinzel, W. and Yeomans, J., J. Phys. A (Math. Gen.) 14 (1981) L 163.

[11] Cardy, J. L. and Sugar, R. L., J. Phys. A (Math. Gen.) 13 (1980) L 423.

[12] Day, A. R. and Lubensky, T. C., J. Phys. A (Math. Gen.) 15(1982).

[13] Redner, S. and YANG, Z. R., J. Phys. A (Math. Gen.) 15 (1982).

[14] Dhar, D., Phani, M. K. and Barma, M., Tata Institute, preprint (1982).

[15] Nadal, J. P., paper presented at the $2^{\text {nd }}$ Statistical Physics Encounter, Paris (January 1982).

[16] Oвuкhov, S. P., Private communication. 